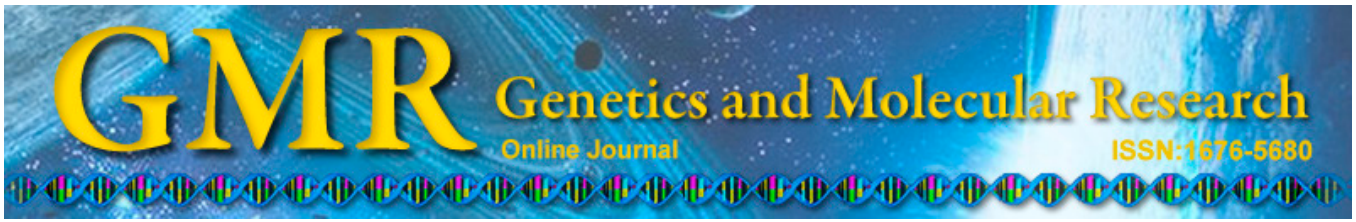

$\underline{\text { Short Communication }}$

\title{
Polymorphisms in GSTM1, GSTT1, GSTP1, and GSTM3 genes and breast cancer risk in northeastern Mexico
}

\author{
G. Jaramillo-Rangel ${ }^{1}$, M. Ortega-Martínez ${ }^{1}$, R.M. Cerda-Flores ${ }^{2}$ and \\ H.A. Barrera-Saldaña ${ }^{3}$ \\ ${ }^{1}$ Departamento de Patología, Facultad de Medicina, \\ Universidad Autónoma de Nuevo León, Monterrey, Nuevo León, México \\ ${ }^{2}$ Facultad de Enfermería, Universidad Autónoma de Nuevo León, \\ Monterrey, Nuevo León, México \\ ${ }^{3}$ Vitagénesis S.A. de C.V., Monterrey, Nuevo León, \\ México and Departamento de Bioquímica y Medicina Molecular, \\ Facultad de Medicina, Universidad Autónoma de Nuevo León, \\ Monterrey, Nuevo León, México \\ Corresponding author: H.A. Barrera-Saldaña \\ E-mail: habarrera@gmail.com
}

Genet. Mol. Res. 14 (2): 6465-6471 (2015)

Received January 9, 2015

Accepted April 29, 2015

Published June 11, 2015

DOI http://dx.doi.org/10.4238/2015.June.11.22

ABSTRACT. Glutathione $S$-transferases (GSTs) are a family of phase II metabolizing enzymes involved in carcinogen detoxification and the metabolism of various bioactive compounds. Several genes that code for these enzymes are polymorphic in an ethnicity-dependent manner, with particular genotypes previously associated with an increased risk of breast cancer. The purpose of this study was to determine the frequencies of polymorphisms in the genes GSTM1, GSTT1, GSTP1, and GSTM3 and to investigate whether an association exists between these genes and breast cancer risk in subjects from northeastern Mexico. Genotypes were determined for 243 women with histologically 
confirmed breast cancer and 118 control subjects. Gene polymorphisms were analyzed using a DNA microarray. We found an increased breast cancer risk associated with the GSTM1 gene deletion polymorphism $(\mathrm{OR}=2.19 ; 95 \% \mathrm{CI}=1.50-3.21 ; \mathrm{P}=0.001)$. No associations between the GSTT1, GSTP1, and GSTM3 genotypes and neoplasia risk were observed. In conclusion, we determined the genotype distribution of GST polymorphisms in control subjects and breast cancer patients from northeastern Mexico. The GSTM1 null genotype was associated with breast cancer risk. Our findings may be used to individualize breast cancer screening and therapeutic intervention in our population, which displays ethnic characteristics that differentiate it from other populations in Mexico.

Key words: Breast cancer; Ethnicity; Glutathione $S$-transferases; Northeastern Mexico; Polymorphisms

\section{INTRODUCTION}

Breast cancer is the most frequently diagnosed malignancy and the leading cause of cancer death in females in both developed and developing countries (Jemal et al., 2011). Although the mechanism of breast carcinogenesis is not fully understood, it is clear that both genetic and environmental factors play a role in this disease. Up to $5 \%$ of all breast cancers arise from germ-line mutations in high-penetrance breast cancer susceptibility genes such as $B R C A 1$ and BRCA2. Therefore, the interaction between polymorphic, low-penetrance genes, and lifestyle or environmental risk factors likely accounts for a much higher proportion of breast cancer cases (Sarmanová et al., 2004).

Low-penetrance genes can participate in the detoxification of environmental carcinogens, steroid hormone metabolism, and DNA damage repair pathways (Mitrunen and Hirvonen, 2003). Glutathione $S$-transferases (GSTs) are a family of phase II metabolizing enzymes involved in carcinogen detoxification and the metabolism of various bioactive compounds (Coles and Kadlubar, 2003). Functional polymorphisms in GSTM1, GSTT1, and GSTP1 have been investigated for their associations with breast cancer in a large number of studies (Egan et al., 2004; Syamala et al., 2008; Van Emburgh et al., 2008; Saxena et al., 2009; Ramalhinho et al., 2011; Reding et al., 2012; Sohail et al., 2013). In contrast, studies analyzing the relationship between GSTM3 and breast cancer are limited (Mitrunen et al., 2001). Identification of inter-individual variability in GST polymorphisms may be useful for individualizing breast cancer screening and therapeutic intervention.

Most studies previously were carried out in Caucasians, African-Americans, and Asians. In Mexico, only one related study has been carried out, in which subjects in the country's central zone were analyzed (Martínez-Ramírez et al., 2013). Thus, the purpose of this study was to investigate the genotypic and allelic frequencies of polymorphisms in GSTM1, GSTT1, GSTP1, and GSTM3 to determine whether an association exists between polymorphisms in these genes and the risk of breast cancer in subjects from northeastern Mexico. 


\section{MATERIAL AND METHODS}

\section{Biological samples}

The patient population and methods have been described in detail in a previous report (Alcazar-González et al., 2013). The case subjects included 243 women with histologically confirmed breast cancer who received chemotherapy at the University Cancer Center of the University Hospital "Dr José E González" of the Autonomous University of Nuevo Leon and the Hospital of Specialities number 25 of the Mexican Institute of Social Security, both located in Monterrey, Nuevo León, Mexico. Both are reference centers for breast cancer patients from throughout the northeastern area of Mexico, including the states of Zacatecas, San Luis Potosí, Tamaulipas, Coahuila, and Nuevo León. A total of 118 controls with no previous history of any type of cancer or other vital disease were also studied. This study conformed to the Declaration of Helsinki, was approved by the local ethics committee (registration HU BI10-002), and all participants provided informed written consent.

\section{Genotyping}

Genomic DNA was obtained from peripheral blood samples either using the QIAamp DNA Blood Mini Kit (Qiagen, Hilden, Germany) following the manufacturer protocol or using TSNT lysis buffer (1\% Triton, $1 \%$ sodium dodecyl sulfate, $100 \mathrm{mM} \mathrm{NaCl}, 10 \mathrm{mM}$ Tris- $\mathrm{HCl}$, $\mathrm{pH} 8.0$, and $1 \mathrm{mM}$ EDTA) followed by phenol-chloroform extraction and ethanol precipitation.

Analysis of gene polymorphisms was performed using the PHARMAchip ${ }^{\circledR}$ DNA microarray following manufacturer protocols (Progenika Biopharma SA, Derio, Spain). Deletion polymorphisms in GSTM1 and GSTT1 and a base transition polymorphism at codon 105 (Ile/ Val) in GSTP1 were analyzed. In the GSTM3 gene, the GSTM3*A wild-type and GSTM ${ }^{*} B$ variant allele, which differed by a deletion of 3 base pairs in intron 6 , were analyzed.

\section{Statistical analysis}

To identify significant differences in polymorphism frequencies between case and control groups, allele and genotype frequencies were compared statistically using the $\mathrm{R} \times \mathrm{C}$ contingency-table exact test. Hardy-Weinberg equilibrium (HWE) for GSTP1 and GSTM3 was estimated using the Chi-square test. HWE was not evaluated for GSTM1 and GSTT1 because the genotyping method did not distinguish heterozygous and homozygous genotypes. The data were input to SPSS, version 22.0 (SPSS Inc., Chicago, IL, USA) for handling and further statistical analyses. ORs and $95 \% \mathrm{CI}$ were calculated to determine significant associations using the Epi Info program (version 7.1.3, CDC, Atlanta, GA, USA). In all analyses, a significance level of 0.05 was adopted.

\section{RESULTS}

Genotypes and allele distributions of GSTM1, GSTT1, GSTP1, and GSTM3 polymorphisms in cases and controls are summarized in Table 1. Thirty-four (30.1\%) of the control subjects had the GSTM1 null genotype. The observed frequency of the null genotype in breast cancer patients was $48.5 \%$. The GSTM1 null genotype was associated with a 2.19 -fold 
$(95 \% \mathrm{CI}=1.50-3.21)$ increase in the risk of developing breast cancer. This association was statistically significant $(\mathrm{P}=0.001)$.

Table 1. Genotype and allele frequencies of glutathione $S$-transferase (GST) genes polymorphisms in breast cancer patients and healthy controls from northeastern Mexico.

\begin{tabular}{|c|c|c|c|c|}
\hline Gene & Frequencies & Cases [N $\left.\mathrm{N}^{\mathrm{a}}(\%)\right]$ & Controls [N $(\%)]$ & $P$ value ${ }^{b}$ \\
\hline GSTMI & $\begin{array}{l}\text { Genotype } \\
\text { Present } \\
\text { Null } \\
\text { Total }\end{array}$ & $\begin{array}{l}124(51.5) \\
117(48.5) \\
241(100)\end{array}$ & $\begin{array}{c}79(69.9) \\
34(30.1) \\
113(100)\end{array}$ & 0.001 \\
\hline GSTTI & $\begin{array}{l}\text { Genotype } \\
\text { Present } \\
\text { Null } \\
\text { Total }\end{array}$ & $\begin{array}{r}211(86.8) \\
32(13.2) \\
243(100)\end{array}$ & $\begin{array}{r}92(80.9) \\
22(19.1) \\
114(100)\end{array}$ & 0.156 \\
\hline GSTP1 & $\begin{array}{l}\text { Genotype } \\
\text { Ile/Ile } \\
\text { Ile/Val } \\
\text { Val/Val } \\
\text { Total } \\
\chi^{2} \\
\text { Allele } \\
\text { Ile } \\
\text { Val }\end{array}$ & $\begin{array}{c}58(24.0) \\
105(43.4) \\
79(32.6) \\
242(100) \\
3.82^{\mathrm{c}} \\
\\
0.4570 \\
0.5430\end{array}$ & $\begin{array}{c}35(29.7) \\
53(44.9) \\
30(25.4) \\
118(100) \\
1.18^{\mathrm{d}} \\
\\
0.5212 \\
0.4788\end{array}$ & 0.301 \\
\hline GSTM3 & $\begin{array}{l}\text { Genotype } \\
{ }^{*} \mathrm{~A} /{ }^{*} \mathrm{~A} \\
*^{\mathrm{A}} / *^{\mathrm{B}} \\
\text { *B/*B } \mathrm{B} \\
\text { Total } \\
\chi^{2} \\
\text { Allele } \\
\text { *A }^{2} \\
\text { *B }\end{array}$ & $\begin{array}{c}198(83.9) \\
34(14.4) \\
4(1.7) \\
236(100) \\
2.93^{\mathrm{d}} \\
\\
0.9110 \\
0.0890\end{array}$ & $\begin{array}{c}102(87.2) \\
15(12.8) \\
0(0.0) \\
117(100) \\
0.54^{\mathrm{d}}\end{array}$ & 0.329 \\
\hline
\end{tabular}

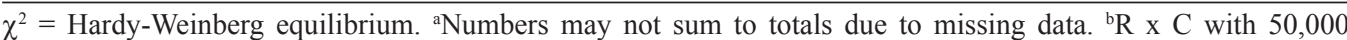
simulations. ${ }^{\mathrm{c}} \mathrm{P}=0.05 .{ }^{\mathrm{d}} \mathrm{P}>0.05$

The GSTT1 null genotype was observed in 13.2 and $19.1 \%$ of cases and controls, respectively. In all, $43.4 \%$ of cases and $44.9 \%$ of controls were heterozygous and $32.6 \%$ of cases and $25.4 \%$ of controls were homozygous for the GSTP1 Ile105Val polymorphism. Finally, when the GSTM3 gene was analyzed, $14.4 \%$ of cases and $12.8 \%$ of controls carried the heterozygous $* \mathrm{~A} / * \mathrm{~B}$ genotype, while $1.7 \%$ of cases and $0.0 \%$ of controls carried the homozygous $* \mathrm{~B} /{ }^{*} \mathrm{~B}$ genotype. The distribution of genotype frequencies of GSTP1 and GSTM3 were in HWE, with the exception of GSTP1 frequencies in breast cancer cases. No associations between GSTT1, GSTP1, and GSTM3 genotypes and breast cancer risk were observed.

\section{DISCUSSION}

Enzymes involved in carcinogen detoxification and metabolism of bioactive compounds have been shown to be polymorphic in an ethnicity-dependent manner. In the present study, genetic polymorphisms of GSTs were investigated in subjects from northeastern Mexico, and their possible association with breast cancer risk was evaluated.

The genotype distributions for GSTM1, GSTT1, and GSTP1 polymorphisms in control subjects were in agreement with the results of previous reports in Mexicans subjects (Pérez- 
Morales et al., 2011; Martínez-Rodríguez et al., 2013). The frequency of the GSTM1 null genotype found in this study was $30 \%$, which is similar to the values observed in Asians and African Americans ( $27 \%)$, but not similar to the value in Caucasians $(\sim 50 \%)$. The frequencies of the Ile/Ile (29\%) and Val/Val (25\%) genotypes of GSTP1 found in this study were more similar to those in African Americans ( $\sim 29$ and $\sim 20 \%$, respectively) that in Caucasians ( $\sim 44$ and $\sim 10 \%$ ) and Asians ( $\sim 99$ and $\sim 7 \%)$. The GSTT1 null genotype frequency (19\%) was similar to that in most populations analyzed thus far (Egan et al., 2004; Syamala et al., 2008; Van Emburgh et al., 2008; Saxena et al., 2009; Ramalhinho et al., 2011; Reding et al., 2012; Sohail et al., 2013). Finally, we determined the frequencies of the GSTM3 genotypes in Mexican subjects. Previous reports regarding GSTM3 genetic polymorphisms frequencies are very limited in the literature (Mitrunen et al., 2001).

A homozygous deletion (null genotype) of GSTM1 or GSTT1 was found to be associated with a lack of the corresponding enzyme activity. The presence of the substitution of isoleucine for valine results in reduced activity of the GSTP1 enzyme. Relatively little is known about the role of GSTM3 in the metabolism of carcinogenic compounds, but the GSTM3 *A and $* B$ alleles may be expressed at different levels with different efficiencies in the metabolism of harmful agents (Mitrunen et al., 2001). This may compromise an individual's ability to deactivate carcinogens, thus increasing the risk of cancer (Mitrunen and Hirvonen, 2003). In the current study, only the GSTM1 null genotype was associated with breast cancer risk. We found no significant increase in breast cancer risk associated with the GSTT1, GSTP1, and GSTM3 genotypes.

A large number of studies have examined the relationship between $G S T$ polymorphisms and breast cancer risk. Overall, no clear pattern has emerged. Studies in which an association between individual or combined GST genotypes and breast cancer was observed were not confirmed by other studies and were refuted (Mitrunen et al., 2001; Egan et al., 2004; Syamala et al., 2008; Van Emburgh et al., 2008; Saxena et al., 2009; Ramalhinho et al., 2011; Reding et al., 2012; Sohail et al., 2013; also see for reviews and meta-analyses: Mitrunen and Hirvonen, 2003; Sergentanis and Economopoulos, 2010; Chen et al., 2011). The discrepancies among studies may be due to several factors, including differences in ethnicity, environmental exposure to carcinogens, and diet. Alternatively, these differences may be attributable to methodological issues, such as the study design, sample size, and analysis method.

In the only previous related study carried out in Mexico, GSTP1 polymorphisms were associated with an increased breast cancer risk; no association was observed for GSTM1 and GSTT1 (Martínez-Ramírez et al., 2013). The differences between this study and our results are likely related to ethnicity and methodological factors.

The admixture among Amerindians, Europeans, and Africans resulted in Mestizos, who represents more than $90 \%$ of the Mexican population (Instituto Nacional de Estadística y Geografía, 2010). However, the Mexican population exhibits high genetic variability. Rubi-Castellanos et al. (2009) analyzed the genetic data of 13 combined DNA index systemshort tandem repeats in subjects representing population samples from different regions of Mexico. They found significant genetic differentiation among Mestizos from different Mexican regions, mainly between the North and the Central and South regions. Furthermore, they observed genetic heterogeneity or asymmetric admixture throughout Mexico, displaying an increasing North-to-South gradient of Amerindian ancestry, and vice versa regarding the European component. Our study examined subjects from northeastern Mexico, whereas in the 
previous study, subjects of the country's central zone were analyzed. Thus, ethnic diversity may have contributed to the discrepancies observed between these studies.

However, the differences in results between both studies may be related to methodological issues. In our study, the genotype frequencies of GSTP1 deviated from the HWE in the case group (Table 1). Because other polymorphisms did not deviate from HWE, our sample set was appropriately ascertained (Wittke et al., 2005). Deviation from HWE may have resulted from random selection, likely because of sample size. Thus, an analysis of a larger sample size of our population may reveal an association between the GSTP1 genotype and breast cancer risk, which would coincide with the results of the other study carried out in Mexico.

In conclusion, we determined the genotype distribution of GSTM1, GSTT1, GSTP1, and GSTM3 polymorphisms in control subjects and breast cancer patients from northeastern Mexico. Only the GSTM1 null genotype was associated with breast cancer risk. Analysis of a larger sample, inclusion of matched controls, and the consideration of gene-environment interactions could augment our understanding of the association between GSTs and breast cancer risk in our population.

\section{REFERENCES}

Alcazar-González GA, Calderón-Garcidueñas AL, Garza-Rodríguez ML, Rubio-Hernández G, et al. (2013). Comparative study of polymorphism frequencies of the CYP2D6, CYP3A5, CYP2C8 and IL-10 genes in Mexican and Spanish women with breast cancer. Pharmacogenomics 14: 1583-1592.

Chen XX, Zhao RP, Qiu LX, Yuan H, et al. (2011). Glutathione S-transferase T1 polymorphism is associated with breast cancer susceptibility. Cytokine 56: 477-480.

Coles BF and Kadlubar FF (2003). Detoxification of electrophilic compounds by glutathione S-transferase catalysis: determinants of individual response to chemical carcinogens and chemotherapeutic drugs? Biofactors 17: 115-130.

Egan KM, Cai Q, Shu XO, Jin F, et al. (2004). Genetic polymorphisms in GSTM1, GSTP1, and GSTT1 and the risk for breast cancer: results from Shanghai Breast Cancer Study and meta-analysis. Cancer Epidemiol. Biomarkers Prev. 13: 197-204.

Instituto Nacional de Estadística y Geografía. Censo de Población y Vivienda. [www.censo2010.org.mx]. Accessed October 24, 2014.

Jemal A, Bray F, Center MM, Ferlay J, et al. (2011). Global cancer statistics. CA Cancer J. Clin. 61: 69-90.

Martínez-Ramírez OC, Pérez-Morales R, Castro C, Flores-Díaz A, et al. (2013). Polymorphisms of catechol estrogens metabolism pathway genes and breast cancer risk in Mexican women. Breast 22: 335-343.

Mitrunen K and Hirvonen A (2003). Molecular epidemiology of sporadic breast cancer. The role of polymorphic genes involved in oestrogen biosynthesis and metabolism. Mutat. Res. 544: 9-41.

Mitrunen K, Jourenkova N, Kataja V, Eskelinen M, et al. (2001). Glutathione S-transferase M1, M3, P1 and T1 genetic polymorphisms and susceptibility to breast cancer. Cancer Epidemiol. Biomarkers Prev. 10: 229-236.

Pérez-Morales R, Méndez-Ramírez I, Castro-Hernández C, Martínez-Ramírez OC, et al. (2011). Polymorphisms associated with the risk of lung cancer in a healthy Mexican Mestizo population: application of the additive model for cancer. Genet. Mol. Biol. 34: 546-552.

Ramalhinho AC, Fonseca-Moutinho JA and Breitenfeld L (2011). Glutathione S-transferase M1, T1, and P1 genotypes and breast cancer risk: a study in a Portuguese population. Mol. Cell. Biochem. 355: 265-271.

Reding KW, Chen C, Lowe K, Doody DR, et al. (2012). Estrogen-related genes and their contribution to racial differences in breast cancer risk. Cancer Causes Control 23: 671-681.

Rubi-Castellanos R, Martínez-Cortés G, Muñoz-Valle JF, González-Martín A, et al. (2009). Pre-Hispanic Mesoamerican demography approximates the present-day ancestry of Mestizos throughout the territory of Mexico. Am. J. Phys. Anthropol. 139: 284-294.

Sarmanová J, Sůsová S, Gut I, Mrhalová M, et al. (2004). Breast cancer: role of polymorphisms in biotransformation enzymes. Eur. J. Hum. Genet. 12: 848-854.

Saxena A, Dhillon VS, Raish M, Asim M, et al. (2009). Detection and relevance of germline genetic polymorphisms in glutathione S-transferases (GSTs) in breast cancer patients from northern Indian population. Breast Cancer Res. Treat. 115: 537-543. 
Sergentanis TN and Economopoulos KP (2010). GSTT1 and GSTP1 polymorphisms and breast cancer risk: a metaanalysis. Breast Cancer Res. Treat. 121: 195-202.

Sohail A, Kanwal N, Ali M, Sadia S, et al. (2013). Effects of glutathione-S-transferase polymorphisms on the risk of breast cancer: a population-based case-control study in Pakistan. Environ. Toxicol. Pharmacol. 35: 143-153.

Syamala VS, Sreeja L, Syamala V, Raveendran PB, et al. (2008). Influence of germline polymorphisms of GSTT1, GSTM1, and GSTP1 in familial versus sporadic breast cancer susceptibility and survival. Fam. Cancer 7: 213-220.

Van Emburgh BO, Hu JJ, Levine EA, Mosley LJ, et al. (2008). Polymorphisms in CYP1B1, GSTM1, GSTT1 and GSTP1, and susceptibility to breast cancer. Oncol. Rep. 19: 1311-1321.

Wittke-Thompson JK, Pluzhnikov A and Cox NJ (2005). Rational inferences about departures from Hardy-Weinberg equilibrium. Am. J. Hum. Genet. 76: 967-986. 